\title{
EL MODELO MAC SOBRE ABURRIMIENTO SEGÚN WESTGATE
}

\section{The MAC model about boredom according to westgate}

Arturo Solf Zárate Universidad Nacional Mayor de San Marcos, Perú

\begin{abstract}
Resumen
Se presentan las características básicas del modelo MAC (Meaning and AttentionalComponents) sobre el aburrimiento según Westgate (2018), su utilidad para la labor del psicólogo, enfatizándose la posibilidad de aplicarlo a cualquier contexto. Asimismo se presenta la fundamentación teórica e investigación básica y sus vínculos con algunos enfoques e intervenciones de otras áreas de la psicología, completándose con algunas propuestas sobre futuras investigaciones que se pueden propiciar teniendo como referente el modelo. Finalmente, se analiza una reacción colectiva de ciudadanos como expresión pública de protesta.
\end{abstract}

Palabras clave: Aburrimiento, modelo MAC, Erin Westgate, motivación laboral, estrés, trabajo significativo.

\begin{abstract}
The basic characteristics of the MAC (Meaning and Attentional Components) model about boredom according to Westgate (2018) are presented. Its usefulness for the work of the psychologist. The possibility to apply it to any context. What is its theoretical foundation and basic research. Its links with some approaches and interventions in other areas of psychology. What future research can be facilitated with the model as a reference. Finally, it is used to analyze a collective reaction of citizens as a public expression of protest
\end{abstract}

Keywords: Boredom, MAC model, Erin Westgate, work motivation, stress, significant work.

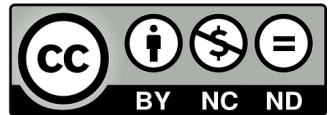

Cualquier uso que se haga de este artículo debe incluir: Autor / Título original de la publicación / ISSN.

\footnotetext{
* Psicólogo. arturo.solf@gmail.com. https://orcid.org/ 0000-0003-3238-3504
} 


\section{INTRODUCCIÓN}

El aburrimiento como una emoción negativa que las personas pueden experimentar al participar activa o pasivamente en un contexto determinado, es motivo de mucha investigación y análisis actualmente. El presente trabajo divulga uno de los aportes más recientes sobre el tema, el modelo MAC [Meaning and Attentional Components] elaborado por Erin Westgate (2018).

Se presentan las características básicas del modelo MAC (Meaning and AttentionalComponents) sobre el aburrimiento según Westgate (2018), su utilidad para la labor del psicólogo, enfatizándose la posibilidad de aplicarlo a cualquier contexto. Asimismo se presenta la fundamentación teórica e investigación básica y sus vínculos con algunos enfoques e intervenciones de otras áreas de la psicología, completándose con algunas propuestas sobre futuras investigaciones que se pueden propiciar teniendo como referente el modelo. Finalmente, se analiza una reacción colectiva de ciudadanos como expresión pública de protesta.

En el documento se ha evitado incidir en aspectos vinculados a la definición de aburrimiento o su medición respectiva, los que pueden ser consultados en otras fuentes (p.ej., Solf, 2018).

\section{MODELO MAC SOBRE EL ABURRIMIENTO}

La psicóloga americana Erin Westgate ha realizado investigaciones sobre el aburrimiento, las que le sirvieron para obtener el doctorado en psicología y proponer el modelo MAC, el cual integra otros enfoques previamente existentes al respecto (Westgate, 2018; Westgate y Wilson, 2018).

El modelo MAC señala que existen dos dimensiones independientes que pueden originar aburrimiento en las personas: atención y significado.

Las personas con bajo aburrimiento se caracterizan por concentrarse en la realización de tareas (atención) que permitan el logro de sus objetivos valiosos (significado). Mientras que, estarán aburridas de presentar deficiencias en la atención y/o en el significado.
BAJO ABURRIMIENTO = ATENCIÓN + SIGNIFICATIVAD

El MAC identifica tres tipos básicos de aburrimiento:

1. Aburrimiento atencional: Originado por la dificultad para mantener una actividad continua en la realización de una tarea específica.

2. Aburrimiento por significado: Se presenta el aburrimiento cuando la persona no percibe algún valor a la tarea realizada. No considera que está vinculada a algún objetivo valioso o importante

3. Aburrimiento mixto: Se activa el aburrimiento al existir dificultades para concentrarse en una tarea no valorada por la persona.

\section{¿Qué influye para la atención?:}

El nivel de atención que las personas le otorgarán a una determinada actividad dependerá de lo siguiente:

a) La congruencia entre las demandas de la tarea (Ds) y los recursos de la persona (Rs). Las Ds tienen que ver con el nivel de dificultad, cantidad, variedad, y exigencia de las labores. Los Rs, están vinculados a la experiencia, formación, y especialmente a los aspectos cognitivos de las personas. Se espera bajo aburrimiento cuando las Ds = Rs (p.ej., altas Ds laborales y altos Rs personales; bajas Ds laborales y bajos Rs personales).

Se activará el aburrimiento en los casos que exista incongruencias entre Ds y Rs: las Ds laborales son mayores que los Rs personales (sobrestimulación) o las Ds laborales son menores que los Rs personales (subestimulación).

CONGRUENCIA: Nivel Ds $=$ Nivel Rs $\quad($ No aburrimiento $)$ INCONGRUENCIA: Ds $>$ Rs / Ds $<$ Rs (Aburrimiento)

b) Características medioambientales del trabajo. En qué medida las condiciones del trabajo, facilita o impide la atención, p. ej., continuas interrupciones, sonidos, espacios físicos reducidos, etc. 


\section{¿Qué influye para la significatividad?}

El personal otorgará determinado valor a sus tareas según diferentes factores tales como: en qué medida la tarea es un medio para lograr un objetivo valioso (p.ej., reconocimiento), la ejecución de la tarea es intrínsecamente agradable para él, cuánto valor le otorgan los demás a la tarea, grado de conocimiento de los efectos de su labor en los demás, congruencia entre la tarea y sus principales valores.

\section{UTILIDAD DEL MODELO MAC PARA EL PSICÓLOGO}

- En la realización de diagnósticos con fines de intervención sería necesario obtener respuestas sobre: ¿Cuán significativo es para el personal su trabajo? ¿Las demandas laborales y los recursos del personal están al mismo nivel? ¿Qué facilidades u obstáculos existen en el contexto de trabajo?

- Reconocer que el aburrimiento no está necesariamente vinculado solo a tareas simples y rutinarias. También puede presentarse en un trabajo con mucha actividad y poco tiempo disponible, por ejemplo.

- Indagar en todos los cargos cuán significativo es la labor para el trabajador, aun para actividades rutinarias y simples.

- Según los déficits detectados la intervención y asesoría variará. En el caso de existir problemas a nivel de congruencia entre Ds laborales y Rs personales podría enfocarse de la siguiente manera:

- Regular las demandas, para conseguir un incremento o disminución de las exigencias en búsqueda de un equilibrio. Mucho dependerá de las competencias del trabajador para persuadir y/o negociar con jefes y colegas en favor de los cambios necesarios.

- Regular los recursos, especialmente los cognitivos. Los recursos se pueden incrementar con ciertas acciones (p.ej., capacitación, realizar prácticas, coaching, meditación, ingiriendo café). En el caso que la persona posee más recursos de lo necesario para su trabajo (Ds $<$ Rs) se podría considerar acciones como: utilizar sus capacidades cognitivas en labores que ayuden a los demás, como puede ser capacitar a un nuevo trabajador o colaborar con un colega en ciertas tareas; luego de concluir sus labores utilice sus recursos cognitivos en variadas actividades en su contexto familiar. La idea central en este caso, es que el personal encuentre oportunidades para utilizar su potencial cognitivo en el trabajo o fuera de éste.

- El cambio de tarea también constituye una opción factible según el caso.

- Si el problema es sobre significatividad se podría superar al otorgarle retroinformación al personal sobre los efectos de su trabajo en las demás áreas. Reflexione sobre las posibles consecuencias para la empresa, en el caso hipotético que eliminen sus funciones. Identifique qué aprendizajes ha obtenido en base a sus actividades. Exprese en qué circunstancias se ha sentido orgulloso al realizar sus labores. Indique cuánto aprecian su trabajo la familia.

\section{APLICACIÓN DEL MODELO MAC A DIFERENTES CONTEXTOS}

El modelo MAC es aplicable a cualquier contexto además del ámbito organizacional. Por ejemplo, en el área educativa sería necesario interrogar: ¿El alumno considera útil e interesante lo que enseña su profesor? ¿Lo que enseña el profesor es muy difícil o muy fácil para el alumno? ¿Las condiciones del contexto son adecuadas (p.ej., espacio físico, acústica, cantidad de alumnos, calidad de los materiales)?

Durante el confinamiento obligatorio de la población como consecuencia de la pandemia del covid-19, podemos describir algunas conductas desde la perspectiva del modelo MAC:

- El profesor cuya actividad docente siempre fue presencial y ahora tiene que dictar clase por internet, pero no es muy diestro para el uso de los medios virtuales (Ds $>$ Rs) y se aburre 
en cada clase a pesar que le agrada enseñar (significatividad).

- Una trabajadora que por internet se inscribe en un curso sobre tejido, y tiene experiencia previa para esta actividad (Ds = Rs) y se siente feliz por los elogios que le proporcionan la profesora y sus familiares conforme avanza su tarea (significatividad).

- El jefe en una empresa que está muy identificado con su organización (significatividad), pero su departamento es pequeño y sus 4 hijos le interrumpen mucho (baja atención), como consecuencia está muy aburrido e irritable.

- Una pareja de esposos que están acostumbrados a labores simples y rutinarias (Ds $=\mathrm{Rs}$ ) se dedican a elaborar diferentes comidas que son mostradas en su página web, para luego recibir elogios (significatividad).

- Dos esposos que poseen bajos niveles de creatividad y no se les ocurre como utilizar sus capacidades cognitivas de manera novedosa (Ds > Rs). Como consecuencia se incrementa el nivel de aburrimiento, así como las agresiones mutuas y han decidido divorciarse.

- Un trabajador que realiza labores de oficina. En su juventud estuvo interesado en seguir filosofía, pero no lo realizó por razones económicas. Ahora se siente una persona feliz (significatividad) al poder leer en internet a los grandes filósofos (Ds = Rs).

- Dos amigos que provienen de provincias y comparten la vivienda; laboran en diferentes empresas realizando una continua actividad física. Conforme se extiende el periodo de confinamiento se sienten aburridos por la falta de actividad (Ds < Rs). Descubren que la ingestión de alcohol disminuye temporalmente el aburrimiento, convirtiéndose en una actividad reiterativa que propicia una habituación a la bebida.

\section{FUNDAMENTACIÓN TEÓRICA E INVESTIGACIÓN BÁSICA SOBRE EL MAC}

En el estudio e investigación sobre al aburrimiento se ha identificado tres aproximaciones teóricas (Westgate, 2018):

- Teorías medioambientales sobre el aburrimiento: Ubican las principales causas para el aburrimiento en la poca estimulación externa -p.ej., tareas simples y rutinarias. Otra fuente considerada es la restricción para la acción, como sucede con alumnos que tienen la obligación de permanecer en el salón durante la clase o el trabajador que debe cumplir con un horario establecido.

- Teorías atencionales: El origen del aburrimiento lo sitúan en los procesos cognitivos vinculados a la regulación de la atención en la realización de una determinada tarea.

- Teorías funcionales: Indagan la finalidad que posee el aburrimiento para los individuos que lo vivencian. Se pueden diferenciar tres subteorías al respecto: el aburrimiento es una especie de aviso que la actividad no está permitiendo lograr una meta; desde una perspectiva de costobeneficio, el aburrimiento estaría indicando la necesidad de buscar otra alterativa que permita un mejor uso de sus recursos personales; es una señal que la actividad no está posibilitando el logro de una meta significativa.

El modelo MAC integra los tres enfoques teóricos mencionados, y aporta algunas extensiones no consideradas por éstos. Westage (2018) realizó la integración luego de analizar las teorías e investigaciones vinculadas, identificando fortalezas, límites, y contradicciones. Para probar su modelo efectuó un meta-análisis de 14 investigaciones previamente realizadas algunas por la autora y otras conjuntamente con colegas. Los resultados del análisis permitieron otorgar sustento a las siguientes hipótesis:

- La atención y significado son dos diferentes determinantes del aburrimiento

- Existen diferentes perfiles de aburrimiento 
- Se identifica dos tipos de déficits en la atención: sobreestimulación y subestimulación.

- El modelo MAC ayudó a explicar “(...) no solo cuándo y por qué las personas se aburren con actividades externas, sino también cuándo y por qué se aburren con sus propios pensamientos" (Westgate y Wilson, 2018, p. 697).

Para mayor información sobre las investigaciones que apoyan las conclusiones mencionadas se puede revisar la tesis de Westgate (2018) sustentada en la Universidad de Virginia para optar el doctorado en psicología.

\section{MODELO MAC Y VÍNCULOS CON OTRAS DIMENSIONES PSICOLÓGICAS}

El modelo MAC sobre el aburrimiento presenta vínculos importantes con el ámbito de las teorías motivacionales. Así, tenemos el Engagement Laboral (Schaufeli y Salanova), según el cual las personas engaged mostrarán un alto compromiso con su trabajo a nivel cognitivo, conductual, y afectivo, es decir, una motivación total. Se espera bajo aburrimiento de tener la persona un alto nivel de Engagement (Harju et al., 2016; Schaufeli y Salanova, 2014). Desde otra perspectiva, sería poco probable que se observe un buen Engagement Laboral si la persona tiene déficits de atención y/o significado. Como consecuencia práctica, para las intervenciones desde enfoque del Engagement Laboral se recomienda tener en consideración los aportes del modelo MAC.

Otro vínculo importante del modelo MAC es con la intervención Job Crafting relacionado a las acciones que realiza el personal para conseguir un ajuste entre las demandas del trabajo y sus recursos personales. Esto es, una especie de auto-rediseño de su puesto para lograr un equilibrio entre demandas y recursos (Demorouti y Bakker, 2014). Los aportes del Job Crafting (Slemp, 2017) serían de mucha utilidad para el diagnóstico e intervención frente al aburrimiento del tipo atencional según el modelo MAC.

Indagando el ámbito de la psicología clínica podemos ubicar a las terapias conductuales de tercera generación (Pérez, 2014), destacando entre ellas la Terapia de Aceptación y Compromiso [ACT], la cual pone énfasis en los valores del paciente que permiten otorgar significado a sus acciones, y apoyar así su desempeño y salud mental. Es muy ilustrativo el caso que menciona Hayes et al. (2014) de una paciente que durante muchos años había evitado salir de su domicilio por temor a experimentar pánico, a diferencia de su esposo e hijos que no presentaban dicha dificultad. El terapeuta logró con su intervención que la paciente decida salir de su encierro para ir a diferentes lugares de la ciudad. Se facilitó el cambio al descubrir que valoraba mucho la felicidad de sus hijos. Es bueno agregar que, dicha paciente al salir de su domicilio sentía ansiedad y distrés, pero su meta significativa le permitió asumir el riesgo.

La Terapia de Aceptación y Compromiso puede contribuir con el análisis e intervención en la dimensión significatividad del modelo MAC. Además, la ACT ha influido para la creación del Coaching de Aceptación y Compromiso aplicado al ámbito laboral (Flaxman et al., 2013; Blonna, 2010; Collis, 2013). En relación al significado también se dispone aportes de otros especialistas como Steger (2017) quien ha analizado ampliamente la creación de significado y propósito en el trabajo.

\section{FUTURAS INVESTIGACIONES EN BASE AL MODELO MAC}

A partir del modelo MAC se ha sugerido la realización de investigaciones para esclarecer aspectos como (Westgate y Wilson, 2018; Westgate y Steidle, 2020):

- Averiguar por qué las personas no utilizan estrategias que están a su alcance para enfrentar el aburrimiento.

- Indagar la relación entre el aburrimiento como rasgo de personalidad - aburrimiento crónico independiente del contexto- y el aburrimiento como estado -según el contexto y situación de la persona.

- Investigar el aburrimiento desde una perspectiva dinámica. Una persona puede experimentar un tipo determinado de aburrimiento, pero de cambiar el contexto y/o ciertos estados mentales 
puede modificarse en otro tipo de aburrimiento o darse el caso que desaparezca dicha emoción negativa.

- Incluir en las investigaciones otras variables que hasta la fecha no se le otorgado la debida importancia, como es la inteligencia. Una persona con alto nivel de inteligencia, se esperaría que ponga más atención a su tarea y le otorgue significado más fácilmente. También podemos agregar otra dimensión psicológica: la creatividad.

- Analizar según el tipo de aburrimiento qué conductas las personas realizan para eliminarlo o disminuirlo. Westgate y Wilson (2018) han identificado dos categorías de respuesta: disfrute e interés. El disfrute se caracteriza por perseguir acciones simples y agradables que no exijan mayor procesamiento de información. El interés estará presente al buscar situaciones complejas que requieran otorgar significado. Los autores proponen que, en caso de presentarse la búsqueda de disfrute las personas en el futuro mostrarán mayor aburrimiento, al compararse dicha conducta como la "comida chatarra" que produce satisfacción a corto plazo en contra del bienestar a largo plazo. Por lo contrario, la búsqueda por interés al preferir situaciones que exijan una mayor abstracción y procesamiento de información, las personas desarrollarían sus procesos mentales que facilitarían afrontar situaciones aburridas.

- Investigar más sobre la aplicación del MAC sobre el aburrimiento de las personas con su pensamiento. La investigación ha demostrado que las personas al divagar en su pensamiento aumentan el aburrimiento. Según el MAC se espera que las personas no se aburrirán con sus pensamientos, en el caso que le otorguen atención y consideren que es valiosa dicha actividad. En un estudio se observó que, de facilitar a las personas su atención -por ejemplo, disponer de una guía sobre los pensamientos positivos a tener- disminuye el aburrimiento (Westgate et al., 2017). De igual manera, Alahmandi et al. (2017) y Wilson et al. (2019) encontraron que, las personas motivadas para disfrutar intencionalmente de sus pensamientos mostraron menos aburrimiento.

- Indagar la relación entre las dimensiones de atención y significado. ¿En qué circunstancias se influyen entre sí? ¿uno es más importante que el otro?

- Utilizar el modelo MAC en investigaciones sobre el aburrimiento en niños, adolescentes, y mayores de edad (Westgate, 2019).

\section{COMENTARIOS Y RECOMENDACIÓN FINAL}

En el presente trabajo se ha divulgado el modelo MAC sobre el aburrimiento desde la perspectiva de la psicología. Actualmente, dicha dimensión es motivo de mucha investigación en diferentes profesiones además de la psicología, como es el caso de la sociología (Ohlmeier et al., 2020).

$\mathrm{Si}$ bien el modelo MAC principalmente se ha aplicado hasta la fecha en relación a conductas individuales, pensamos que puede ser útil para entender mejor conductas grupales y manifestaciones colectivas, como las recientes protestas públicas de la población- mayormente jóvenes- por la vacancia del presidente del Perú (Martín Vizcarra) decidido por el Congreso de la República. Antes de dicha respuesta, en el país se había dispuesto un prolongado confinamiento obligatorio de los ciudadanos como una medida de protección frente a la presencia del covid-19. Podemos suponer que en la mayoría de los ciudadanos se habría originado niveles altos de aburrimiento y estrés, al no estar acostumbrados a permanecer mucho tiempo en sus hogares, con limitaciones para las relaciones con amigos y familiares, agregándose a ello la pérdida de trabajo o ambigüedades sobre éste. Utilizando el modelo MAC estaríamos frente a un tipo de aburrimiento atencional por desequilibrio (Ds > Rs) a pesar que el confinamiento pueda haber sido considerado necesario para preservar la salud (significado).

Continuando con el análisis de dicha manifestación, es bueno centrarse en las posibles acciones que emprenden las personas para superar la vivencia aversiva del aburrimiento. Según algunas 
investigaciones se ha observado que las personas aburridas tienden a ser impulsivas y asumen más riesgos (Wegner y Flisher, 2009; Kilic et al. 2019; Moynihan et al.,2017), y sus actitudes políticas adoptan niveles más extremos (Van Tilburg y Igout, 2017). De existir en la ciudadanía dicho trasfondo actitudinal, y además una percepción de estar afectándose ciertos valores (p.ej.,"dignidad”, “justicia”, “igualdad,“ participación”), entonces tendríamos las condiciones psicológicas básicas para motivar una expresión masiva y enérgica.

Resumiendo, en el caso analizado es muy probable que haya existido un desequilibrio entre demandas y recursos personales (aburrimiento atencional) y el menoscabo -real o percibido- de valores importantes. Además de estas dimensiones básicas también se puede identificar otras, tales como: ideología política, expectativas hacia el futuro, posición social, inserción en el ámbito laboral, creencias religiosas e identidad generacional. El aburrimiento puede haber servido como un activador e intensificador de la respuesta colectiva

Finalmente, recomendamos que los psicólogos de las diferentes especialidades le otorguen el debido interés al aburrimiento de las personas, al ser un poderoso motivador de la conducta que puede discurrir por caminos positivos o negativos, frente al cual la intervención del profesional puede ser decisiva para evitar lo negativo.

\section{REFERENCIAS}

Alahmandi, S., Buttrick, N. R., Gilbert, D.T., Hardin, A. M., Westgate, E.C., \& Wilson, T. D. (2017). You can do it if you really try: The effects of motivation on thinking for pleasure. [Puedes hacerlo si realmente lo intentas: Los efectos de la motivación en pensar por placer. Motivación y emoción]. Motivation and Emotion,41, 545-561.

Blonna, R. (2010). Maximize your coaching effectiveness with Acceptance \& Commitment Therapy. [Maximice la eficacia de su coaching con la terapia de aceptación y compromiso]. New Harbinger Publications
Collis, R. (2013).Applying ACT to Worplace Coaching: A guide to using Acceptance and Commitment Training in work related coaching. [Aplicando $\mathrm{ACT}$ al coaching en el lugar de trabajo: Una guía para utilizar el entrenamiento de aceptación y compromiso en el coaching relacionado con el trabajo]. Rachel Collis

Demerouti, E., \& Bakker, A. B. (2014). Job Crafting. In Peeters,J. de Jonge, \& T. Taris (Eds). An Introduction to Contemporary Work Psychology, (pp. 414-433). Wiley Blackwel

Flaxman, P.E., Bond, F.W., \& Livheim, F. (2013). The Mindful and Effective Employee: An Acceptance \& Commitment Therapy Training Manual for improving Well-Being and Performance. [El empleado consciente y eficaz: Manual de aceptación y compromiso para mejorar el bienestar y el desempeño]. New Harbinger Publications

Harju, L.K., Hakanen, J.J, \& Schaufeli, W.B. (2016). Can job crafting reduce job boredom and increase work engagement? A three-year cross-lagged panel study. Journal of Vocational Behavior, 9597, 11-20

Hayes, S., Strosahl, K., \& Wilson, K.G. (2014). Terapia de Aceptación y Compromiso: Proceso y práctica del cambio consciente (Mindfulness). Desclée de Brouwer

Kilic, A., van Tilburg., W.A.R., Igou, E.R. (2019). Risk-taking increases under boredom. Journal of Behavioral Decision Making, 33 (3), 257-269

Moynihan, A.B., Igou, E.R., \& van Tilburg, W. A. P. (2017). Boredom increases impulsiveness: A meaning-regulation perspective. Social Psychology, 48 (5), 293-309

Ohlmeier, S.,Finkielsztein, M., \& Paff,H. (2020). Why we are bored: Towards a sociological approach to boredom. Sociological Sprectum. https://doi.org/1 0.1080/02732173.2020.1753134

Pérez, M. (2014). Las Terapias de tercera generación como terapias contextuales. Síntesis

Schaufeli, W.B., \& Salanova, M. (2014). Burnout, Boredom and Engagement in the Workplace. In M. Peeters, J. de Jonge, \& T. Taris (Eds). An Introduction to Contemporary Work Psychology, (pp. 293-320). Wiley Blackwel 
Slemp, G. (2017). Job Crafting. In L.G. Oades, M.F. Steger, A. Delle, \& J. Passmore (Eds), The Wiley Blackwell Handbook of the Psychology of Positive and Strenghs-based approach at Work, (pp.342365). Wiley Blackwell

Solf, A. (2018). El aburrimiento en el trabajo: Aportes recientes de la Psicología Laboral. Avances en Psicología, Unifé, 26(1), 9-22. https://doi. org/10.33539/avpsicol.2018v26n2.1123

Steger, M.F. (2017). Creating meaning and purpose at work. In L.G. Oades, M.F. Steger, A. Delle, \& J. Passmore (Eds), The Wiley Blackwell Handbook of the Psychology of Positive and Strenghs-based approach at Work, (pp.60-82). Wiley Blackwell

Van Tilburg, W.A.P., \& Igout, E.R. (2017). Going to political extremes in response to boredom. European Journal of Social Psychology. https:// doi.org/10.1002/ejsp.2205

Wegner, L., \& Flisher, A.J. (2009). Leisure boredom and adolescent risk behaviour: A systemactic literatura review. Journal of Child Adolescent Mental Health, 2l(1), 1-28

Westgate, E.R. (2018). Why boredom is interesting. A dissertation for the degree of Doctor of Philosophy. University of Virginia. https://libraetd lib.virginia. edu/public_view/4m90dv97n [Noviembre 2020]
Westgate,E.C., \& Wilson,T.D. (2018). Boring thoughts and bored minds: The MAC model of boredom and cognitive engagement. Psychological Review, $125,689-713$

Westgate, E.R. (2019). Are we bored yet? A lifespan perspective on the MAC Model of boredom and cognitive Engagement. https://psyarxiv. com/4h6et/ [Noviembre 2020]

Westgate, E. C., Wilson; T. D., \& Gilbert, D. T. (2017). With a litte help for our thoughts: Making it easier to think for pleasure. Emotion, 17, 828-839

Westgate, E.C. (2020). Why boredom is interesting. Current Directions in Psychological Science, 29(1), 33-40

Westgate, E.C., \& Steidle, B. (2020). Lost by definition: Why boredom matters for psychology and society. Social and Personality Psychology Compass, 14(11), 12-28

Wilson, T.D., Westgate, E. R., Buttrtick, N. R., \& Gilbert, D.T. (2019). The mind is its own place: The difficulties and benefits of thinking for pleasure. Experimental Social Psychology, 60, 175-221

Fecha de recepción: 7 de noviembre 2020

Fecha de aceptación: 12 de enero 2021 